\title{
POTENTIAL SCATTERING AND THE CONTINUITY OF PHASE-SHIFTS
}

\author{
Jesse Gell-Redman and Andrew Hassell
}

\begin{abstract}
Let $S(k)$ be the scattering matrix for a Schrödinger operator (Laplacian plus potential) on $\mathbb{R}^{n}$ with compactly supported smooth potential. It is well known that $S(k)$ is unitary and that the spectrum of $S(k)$ accumulates on the unit circle only at 1 ; moreover, $S(k)$ depends analytically on $k$ and therefore its eigenvalues depend analytically on $k$ provided they stay away from 1 .

We give examples of smooth, compactly supported potentials on $\mathbb{R}^{n}$ for which (i) the scattering matrix $S(k)$ does not have 1 as an eigenvalue for any $k>0$, and (ii) there exists $k_{0}>0$ such that there is an analytic eigenvalue branch $\mathrm{e}^{2 \mathrm{i} \delta(k)}$ of $S(k)$ converging to 1 as $k \downarrow k_{0}$. This shows that the eigenvalues of the scattering matrix, as a function of $k$, do not necessarily have continuous extensions to or across the value 1 . In particular, this shows that a "micro-Levinson theorem" for non-central potentials in $\mathbb{R}^{3}$ claimed in a 1989 paper of R. Newton is incorrect.
\end{abstract}

\section{Introduction}

In this article, we consider scattering in $\mathbb{R}^{n}$ due to a non-positive potential function, which we call a potential well. We denote the potential $-V(x), V \geq 0$, and assume for simplicity that $V$ is smooth and compactly supported. Recall that the Schrödinger operator $H=\Delta-V$, where $\Delta=-\sum \partial_{x_{i}}^{2}$, has absolutely continuous spectrum on $(0, \infty)$ and may have finitely many eigenvalues on the non-positive real axis. The scattering matrix, $S(k), k>0$, can be defined in terms of the generalized eigenfunctions or scattering solutions for $H$. As is well known, for each smooth function $q_{\text {in }}$ on the sphere, there is a unique solution $u$ to $\left(H-k^{2}\right) u=0$, with $u$ taking the form

$$
u=r^{-(n-1) / 2}\left(\mathrm{e}^{-\mathrm{i} k r} q_{\text {in }}(\omega)+\mathrm{e}^{\mathrm{i} k r} q_{\text {out }}(-\omega)\right)+O\left(r^{-(n+1) / 2}\right),
$$

as $r=|x| \rightarrow \infty[6]$. Here $q_{\text {out }} \in C^{\infty}\left(S^{n-1}\right)$. As a consequence of uniqueness, $q_{\text {out }}$ is determined by $q_{\text {in }}$; the map $q_{\text {in }} \mapsto \mathrm{e}^{\mathrm{i} \pi(n-1) / 2} q_{\text {out }}$ is by definition the scattering matrix $S(k)$. The normalization factor $\mathrm{e}^{\mathrm{i} \pi(n-1) / 2}$ is chosen so that this "stationary" definition of the scattering matrix agrees with time-dependent definitions (see, e.g., $[10,11]$ ), and is such that the scattering matrix for the zero potential is the identity. The scattering matrix $S(k)$ extends to a unitary map from $L^{2}\left(S^{n-1}\right)$ to $L^{2}\left(S^{n-1}\right)$ for each $k>0$.

In this note, we are interested in the eigenvalues of the scattering matrix $S(k)$. As $S(k)$ is unitary, these lie on the unit circle, and they are conventionally denoted $\mathrm{e}^{2 \mathrm{i} \delta_{j}(k)}$ where $\delta_{j}$ is real; the $\delta_{j}$ are called phase shifts. They are determined up to a multiple of $\pi$.

The scattering matrix $S(k)$ for smooth, compactly supported potentials takes the form Id $+A(k)$, where $A(k)$ has a smooth kernel. It follows that $S(k)-$ Id is a compact

Received by the editors December 18, 2011. 
operator for each $k$, and therefore the spectrum of $S(k)$ is discrete except at 1 . Moreover, for non-positive potentials, the spectrum only accumulates "from above", that is, from the upper half plane [11, Theorem 1.7.9]. The scattering amplitude $A(k)$ is analytic in $k$, which implies that the eigenprojections of $S(k)$ vary analytically with $k$, provided the eigenvalue stays away from 1.

The question we address here is whether the eigenvalues can pass through 1 , or more precisely, whether a phase shift $\delta(k)$ that tends upward to $\pi$ as $k \rightarrow k_{0}$ can be extended continuously up to, and even past, $k=k_{0}$.

In the case that $V$ is spherically symmetric, the scattering solutions take the form of a spherical harmonic times a function of $r$, and ODE analysis establishes that the phase shifts are analytic for all $k>0$. Furthermore, Levinson's theorem for central potentials can be used to guarantee that eigenvalues of $S(k)$ do pass through 1.

Levinson's Theorem (central potentials). Given $V(r) \in C_{c}^{\infty}(\mathbb{R})$ and a spherical harmonic $\phi$, let $\alpha(k)$ be the eigenvalue of $\phi$ for the scattering matrix $S(k)$ of $H=\Delta-$ $V(|x|)$, i.e., $S(k) \phi=\alpha(k) \phi$. Let $n$ be the dimension of the subspace of $L^{2}$ eigenvectors of $H$ of the form $u=a(r) \phi$. Then the counterclockwise winding number of $\alpha(k)$ as $k$ goes from $\infty$ to 0 satisfies

$$
-\frac{1}{2 \pi i} \int_{0}^{\infty} \frac{\alpha^{\prime}(k)}{\alpha(k)} d k=n+\nu
$$

where $\nu=1 / 2$ if $\phi \equiv 1$ and there is a half-bound state, and is 0 otherwise.

See Theorem XI.59 of [10] for a proof of this well-known fact in the case $\phi \equiv 1$, and equation (5.15) of [7] for the general case. For a fixed, non-positive, spherically symmetric $V$, the number of bound states of $\Delta-\lambda V$ with angular part $\phi$ grows at least as fast as $c \sqrt{\lambda}$ for some constant $c>0$ (Theorem XIII.9 of [9]), so for such $\lambda$ there are eigenvalues of the scattering matrix which pass continuously through 1 .

By contrast, our main result is that, for an arbitrary $C_{c}^{\infty}$ potential function, phase shifts cannot necessarily be so continued: we give an explicit example of a potential well for which

- the scattering matrix $S(k)$ does not have 1 as an eigenvalue for any $k>0$, and

- there exists $k_{0}>0$ and an eigenvalue branch $\mathrm{e}^{2 \mathrm{i} \delta(k)}$ of $S(k)$ such that $\delta(k) \uparrow \pi$ as $k \downarrow k_{0}$.

Lest this seem bizarre, we mention that the corresponding phenomenon for obstacle scattering has already been observed by Eckmann-Pillet [2]. In both cases, the source of the phenomenon is the same: for a compactly supported perturbation of $\mathbb{R}^{n}$, if 1 is an eigenvalue of $S(k)$ then there is a generalized eigenfunction that outside the perturbation agrees exactly with a generalized eigenfunction of the free Laplacian (which has scattering matrix $S(k)$ equal to the identity, and hence has every eigenvalue 1). See Section 2 for an elementary (and well-known) proof of this fact. This is an extremely restrictive situation, and for certain perturbations one can show that it is not possible.

Our interest in the continuity of phase shifts through multiples of $\pi$, or equivalently in eigenvalues of $S(k)$ through 1 , came from reading a 1989 paper of R. Newton published in Annals of Physics in which, in particular, it is claimed that one can label the phase shifts of the scattering matrix for any $C_{c}^{\infty}$ potential in $\mathbb{R}^{3}$ (actually, 
Newton considers the larger class of bounded potentials with exponential decay) in such a way that they are continuous functions of $k \in(0, \infty)$. This is then used to claim a "micro-Levinson theorem" relating phase shifts at $k=0$ to the nonpositive spectrum of $H$. But it is straightforward to show that for any non-negative $V \in C_{c}^{\infty}\left(\mathbb{R}^{3}\right)$ and $\lambda$ sufficiently large, some phase shift of $-\lambda V$ will approach $\pi$ from below as $k$ approaches some finite positive value $k_{0}$ from above (see Section 5 ), and so Newton's result would imply that the scattering matrix of $-\lambda V$ at energy $k_{0}$ has 1 as an eigenvalue. Our example shows that this is not true, and therefore shows that Newton's claimed theorem is incorrect. Further discussion about Newton's paper is given in Section 6 below.

The scattering matrix for non-positive potentials is not the only family of operators of interest in spectral analysis in which there is a one-sided accumulation of spectrum and where one is interested in eigenvalues approaching the accumulation point. Another example is the Neumann-to-Dirichlet operator $N(k)$ for a smooth bounded domain $\Omega$ in $\mathbb{R}^{n}$ (or more generally a Riemannian manifold with boundary). This operator, defined for complex $k$ (except for $k^{2}$ in the Neumann spectrum of $\Omega$ ), takes $L^{2}(\partial \Omega)$ to $L^{2}(\partial \Omega)$ and maps $f \in C^{\infty}(\partial \Omega)$ to the boundary value of the function $u \in C^{\infty}(\Omega)$ satisfying the Helmholtz equation $\left(\Delta-k^{2}\right) u=0$ and the Neumann boundary condition $d_{n} u=f$. This operator is a pseudodifferential operator of order -1 with positive principal symbol, and therefore has an accumulation of eigenvalues at 0 from above. Eigenvalues of $N(k)$ are monotone increasing in $k$ [3] and, for every Dirichlet eigenvalue $k_{0}^{2}$, there is an eigenvalue $\beta(k)$ of $N(k)$ such that $\beta(k) \uparrow 0$ as $k \uparrow k_{0}$. The Neumann-to-Dirichlet operator is quite closely analogous to the scattering matrix (and the analogue becomes even closer if one considers the Cayley transform of $N(k)$, which is a family of unitary operators defined for every $k>0$ and depending analytically on $k$ ). But there is an important difference between the two cases: in the case of $N(k)$, the eigenvalue branches $\beta(k)$ tending to zero as $k \uparrow k_{0}$ always have a continuous extension to $k_{0}$, with the eigenfunction at $k_{0}$ being the normal derivative of the corresponding Dirichlet eigenfunction on $\Omega$. This can be traced to the fact that the eigenfunction branch corresponding to $\beta(k)$ has a weak limit in $H^{1}(\Omega)$ and thus, thanks to the compact embedding $H^{1}(\Omega) \rightarrow L^{2}(\Omega)$, a strong limit as $k \uparrow k_{0}$, which is necessarily non-zero [1]. By contrast, the generalized eigenfunction branch for the scattering problem $\left(H-k^{2}\right) u=0$ corresponding to a phase shift $\delta(k)$ may have only a weak limit — which may be zero - if $\delta \uparrow \pi$ as $k \downarrow k_{0}$.

\section{Consequences of the scattering matrix having eigenvalue 1}

Suppose that we have a compactly supported perturbation, $H$, of the Laplacian $\Delta$ on $\mathbb{R}^{n}$, such that the scattering matrix $S(k), k>0$, has an eigenvalue equal to 1 . Notice that since $S(k)=\mathrm{Id}+A(k)$, where $A(k)$ has a smooth kernel, the eigenfunction, say $q(\omega)$, is smooth. So there is a generalized eigenfunction $u$ of $H$ having the asymptotics

$$
u=r^{-(n-1) / 2}\left(\mathrm{e}^{-\mathrm{i} k r} q(\omega)+\mathrm{e}^{\mathrm{i} k r} \mathrm{e}^{\mathrm{i} \pi(n-1) / 2} q(-\omega)\right)+O\left(r^{-(n+1) / 2}\right) .
$$

Since the scattering matrix for the zero potential is the identity operator for all $k$, there is also a free generalized eigenfunction $u_{f}$, satisfying $\left(\Delta-k^{2}\right) u_{f}=0$ on $\mathbb{R}^{n}$, 
satisfying

$$
u_{f}=r^{-(n-1) / 2}\left(\mathrm{e}^{-\mathrm{i} k r} q(\omega)+\mathrm{e}^{\mathrm{i} k r} \mathrm{e}^{\mathrm{i} \pi(n-1) / 2} q(-\omega)\right)+O\left(r^{-(n+1) / 2}\right) .
$$

It follows that $u-u_{f}=O\left(r^{-(n+1) / 2}\right)$ near infinity. If outside some large ball in $\mathbb{R}^{n}$ we expand $u-u_{f}$ in spherical harmonics, then we find that the coefficients are functions $j_{l}(r)$ such that $r^{(n-2) / 2} j_{l}(r)$ satisfy Bessel's equation of order $l+(n-2) / 2$, and are $O\left(r^{-3 / 2}\right)$ as $r \rightarrow \infty$. As the only such solutions are identically zero, we find that $u=u_{f}$ outside any ball containing the perturbation. Then applying standard unique continuation theorems, such as [5, Theorem 17.2.6], we find that $u=u_{f}$ outside the support of the perturbation; in the case of potential scattering, this means outside the support of $V$.

It is now straightforward to understand the observation of Eckmann-Pillet that the typical smooth obstacle $\Omega$ in $\mathbb{R}^{n}$, endowed with Dirichlet boundary conditions, will never have 1 as an eigenvalue of its scattering matrix $S(k)$ for any $k>0$. For if a free plane wave $u_{f}$ agrees with a generalized eigenfunction for the exterior domain $\mathbb{R}^{n} \backslash \Omega$, then $u_{f}$ vanishes on $\partial \Omega$. But $u_{f}$ is real analytic, so that would imply that $\partial \Omega$ is contained in the zero set of a non-trivial real analytic function, and of course this is not true for a generic smooth obstacle. (As an example, take any smooth compact obstacle whose boundary contains an open subset of a hyperplane.)

On the other hand, the main result of Eckmann and Pillet was that for each $k_{0}$ such that $k_{0}^{2}$ is a Dirichlet eigenvalue of $\Omega$, there is an eigenvalue branch $\beta(k)$ that tends to 1 as $k \uparrow k_{0}$. This furnishes many examples of cases where an eigenvalue branch tending to 1 does not extend continuously to include the value 1 , i.e., when the accumulation point of the spectrum is reached, the eigenfunction ceases to exist. See [2] for the details.

We introduce some terminology to describe these situations. We shall say that a potential function $-V$ is partially transparent at frequency $k_{0}>0$ if its scattering matrix $S\left(k_{0}\right)$ has an eigenvalue 1 ; almost partially transparent at frequency $k_{0} \geq 0$ if there is an eigenvalue branch $\mathrm{e}^{2 \mathrm{i} \delta(k)}$ for $k>k_{0}$ that tends to 1 as $k \downarrow k_{0}$; and completely non-transparent if the scattering matrix is not partially transparent for any $k$, i.e., if the scattering matrix has no eigenvalue equal to 1 for any $k>0$.

In Section 4, we give an example of a completely non-transparent potential. The example is not in the least bit pathological: it is simply a double well potential, where each well is smooth, compactly supported and spherically symmetric, and the only subtlety is that the ratio between the radii of these wells is required to avoid a countable set of values (which may be dense). We believe that this property of being completely non-transparent is generic for potential wells in $C_{c}^{\infty}\left(\mathbb{R}^{n}\right)$, but we do not attempt to prove this in this note.

\section{Model example on $\ell^{2}$}

We now give an explicit example of a family of operators with an eigenvalue branch that vanishes at an accumulation point. This can be regarded as a model for the way in which an eigenbranch for the scattering matrix can disappear when the eigenvalue hits 1 . This example is illustrative only and is not used in the remainder of the paper. 
Let $e_{j}, j=1,2, \ldots$, denote the standard basis of $\ell^{2}=\ell^{2}(\mathbb{N})$, and let $z_{0}$ be any vector with

$$
\begin{aligned}
\left\langle z_{0}, e_{j}\right\rangle & >0 \text { for all } j, \\
\left\langle z_{0}, e_{j}\right\rangle & =\left\langle z_{0}, e_{k}\right\rangle \Longleftrightarrow j=k, \\
\left\|z_{0}\right\| & \leq 1 .
\end{aligned}
$$

For example, we can take $z_{0}=\frac{1}{2} \sum_{j} e_{j} / j$.

We define a self-adjoint compact operator $T$ on $\ell^{2}$ by

$$
T\left(e_{j}\right):=\left|\left\langle z_{0}, e_{j}\right\rangle\right|^{3} e_{j},
$$

and perturb it by a family of rank one self-adjoint operators parametrized by $k \in \mathbb{R}$, as follows:

$$
T_{k}(z):=T(z)+k\left\langle z_{0}, z\right\rangle z_{0} .
$$

Notice that $T$, and hence $T_{k}$, has spectrum accumulating at 0 , from above only.

We will show that $T_{k}$ has the following properties:

(i) for $k \geq 0, T_{k}$ has only positive eigenvalues;

(ii) for $k<0$, there is exactly one negative eigenvalue $\alpha(k)$ of $T_{k}$, satisfying $k<\alpha(k)<0$;

(iii) $T_{k}$ shares no eigenvalue in common with $T_{0}=T$ for $k \neq 0$, and for all $k$, every eigenvalue of $T_{k}$ is simple.

The upshot is that for $k<0$ there is an eigenvalue $\alpha(k)$ of $T_{k}$ which approaches 0 from below as $k \uparrow 0$, but there is no corresponding zero eigenvalue at $k=0$. By point (iii), there is no possible continuation of $\alpha(k)$ to $k \geq 0$, even allowing a removable singularity at $k=0$ : the eigenvalue branch $\alpha(k)$ simply ceases to exist.

The proofs of these three facts are elementary. For $k \geq 0$, the operator $T_{k}$ is manifestly positive, and for $k<0$, it is manifestly bounded below by $k$. For any $k, T_{k}$ restricted to $\operatorname{span}\left\langle z_{0}\right\rangle^{\perp}$ is equal to $T$, hence it is positive off a codimension 1 subspace. On the other hand

$$
\left\langle T_{k}\left(e_{j}\right), e_{j}\right\rangle=\left|\left\langle z_{0}, e_{j}\right\rangle\right|^{3}+k\left|\left\langle z_{0}, e_{j}\right\rangle\right|^{2},
$$

so for $k<0$ this is negative for large $j$. Thus there is exactly one negative eigenvalue if $k<0$.

To prove (iii), suppose that $\lambda$ is an eigenvalue of both $T$ and $T_{k}$ for $k \neq 0$. All eigenspaces of $T$ are simple, so $\lambda=\left|\left\langle z_{0}, e_{j}\right\rangle\right|^{3}$ for some $j$ and the eigenvector is $e_{j}$. Let $z$ be the eigenvector of $T_{k}$. Then, we have

$$
\lambda\left\langle z, e_{j}\right\rangle=\left\langle T_{k} z, e_{j}\right\rangle=\left\langle z, T_{0} e_{j}\right\rangle \Longrightarrow\left\langle\left(T_{k}-T_{0}\right) z, e_{j}\right\rangle=0 .
$$

This means that

$$
k\left\langle z_{0}, z\right\rangle\left\langle z_{0}, e_{j}\right\rangle=0 .
$$

Since $\left\langle z_{0}, e_{j}\right\rangle \neq 0$ we find that

$$
\left\langle z_{0}, z\right\rangle=0
$$




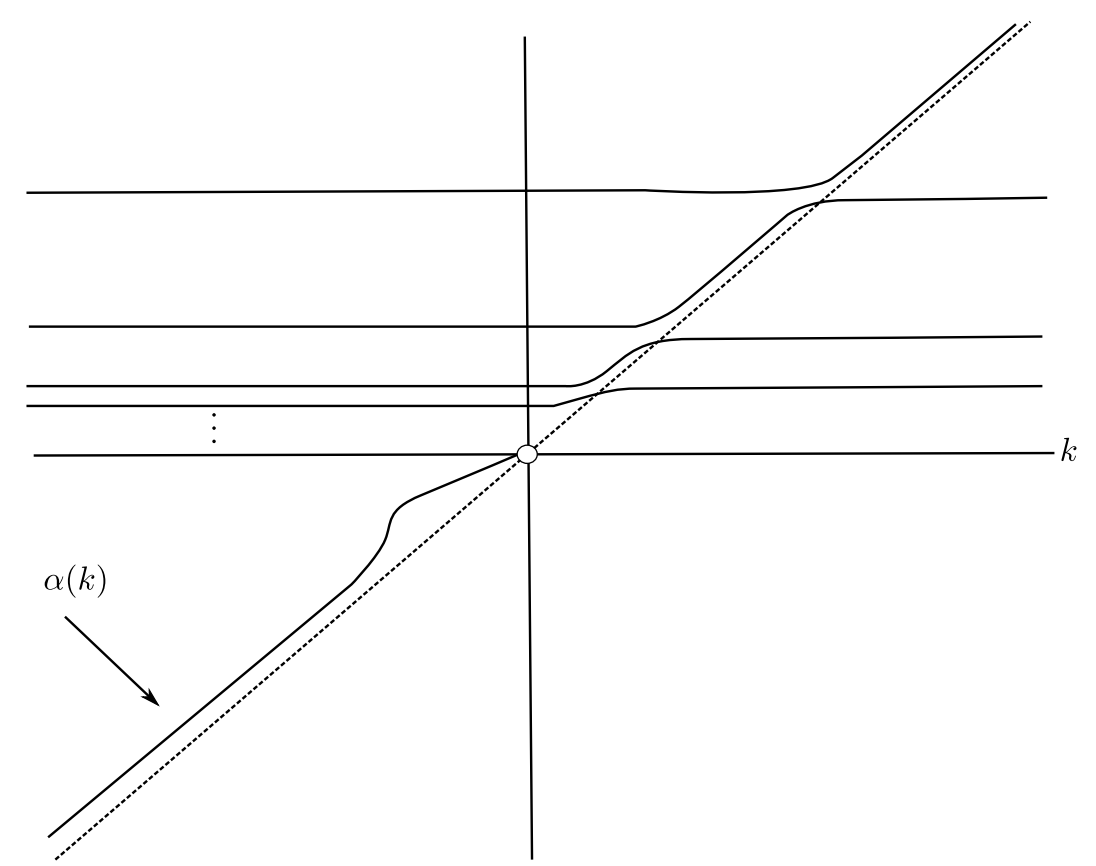

FiguRE 1. Schematic depiction of the spectrum of $T(k)$. The negative eigenvalue branch, $\alpha(k)$, vanishes as $k=0$, the other eigenvalues avoid crossing, and the largest eventually increases as $\left\|z_{0}\right\|^{2} k+O(1)$. The dotted line is the line $y=\left\|z_{0}\right\|^{2} k$.

However, this implies that $T_{k} z=T_{0} z$. Therefore, $z$ is an eigenfunction of $T_{0}$, i.e., $z=e_{i}$ for some $i$. But this contradicts (3.4), since $\left\langle z_{0}, e_{i}\right\rangle \neq 0$. Finally we show that $T_{k}$ has only simple eigenvalues. This is true by construction for $k=0$. For $k \neq 0$, if $T_{k}$ had an eigenspace of two or more dimensions then it would contain a non-trivial eigenvector $w$ orthogonal to $z_{0}$. But then, as before, we would have $T_{k} w=T_{0} w$ so $w$ would be an eigenvector of $T_{0}$, contradicting the fact just proved that $T_{k}$ and $T_{0}$ have no eigenvalues in common.

The spectrum of $T_{k}$ as a function of $k$ therefore is as in figure 1 , with necessarily an infinity of avoided crossings due to property (iii) above.

\section{Completely non-transparent potential}

We now give an example of a compactly supported, smooth potential $V>0$ which is completely non-transparent. In fact, we show a slightly stronger result; for any sequence of "strengths", i.e., positive real numbers $\lambda_{i} \rightarrow \infty$, we find a $V$ such that all of the potentials $-\lambda_{i} V$ are completely non-transparent.

Given any $W \in C_{c}^{\infty}\left(\mathbb{R}^{n}\right)$, let $S_{W}(k)$ denote the scattering matrix of $\Delta-W$. If $W$ is partially transparent at frequency $k$, then, as discussed in Section 2 , there are solutions

$$
\begin{array}{r}
\left(\Delta-W-k^{2}\right) u=0 \\
\left(\Delta-k^{2}\right) u_{f}=0
\end{array}
$$


such that $u=u_{f}$ on the complement of $\operatorname{supp} W$. Let $\Omega$ be any smooth bounded domain containing the interior of $\operatorname{supp} W$. Then, at the boundary of $\Omega$,

$$
\begin{aligned}
\left.u\right|_{\partial \Omega} & =\left.u_{f}\right|_{\partial \Omega}, \\
\left.\partial_{\nu} u\right|_{\partial \Omega} & =\left.\partial_{\nu} u_{f}\right|_{\partial \Omega} .
\end{aligned}
$$

We will construct a domain so that for any functions $u$ and $u_{f}$ satisfying (4.1) on $\Omega$, equation (4.2) holds only when $u \equiv u_{f} \equiv 0$.

Let $\chi \in C_{c}^{\infty}(\mathbb{R})$ denote be a bump function with $\chi \geq 0, \chi(r) \equiv 1$ for $r<1 / 4$, and $\operatorname{supp} \chi \subset\{r \leq 1 / 2\}$. Also choose $x_{0} \in \mathbb{R}^{n}$ with $\left|x_{0}\right|>1$ and $R>0$ such that the intersection $\overline{B_{0}(1)} \cap \overline{B_{x_{0}}(R)}$ is empty, that is, so that $R<\left|x_{0}\right|-1$. We define a potential

$$
V_{R}(x):=\chi(|x|)+R^{-2} \chi\left(\left|x-x_{0}\right| / R\right) .
$$

We think of $x_{0}$ as fixed throughout.

Theorem 1. Let $\Omega_{R}=B_{0}(1) \cup B_{x_{0}}(R)$ where $\left|x_{0}\right|>1$. Then for any sequence of positive numbers $\lambda_{i} \rightarrow \infty$, there is a countable set $\Lambda$ so that for $R \notin \Lambda$ and $0<R<\left|x_{0}\right|-1$, there are no non-zero simultaneous solutions to (4.1) and (4.2) with $W=\lambda_{i} V_{R}$. Thus, for each $\lambda_{i}$, the scattering matrix for $S_{\lambda_{i} V_{R}}(k)$ does not have 1 as an eigenvalue for any $k>0$, i.e., $-\lambda_{i} V_{R}$ is completely non-transparent.

Before we begin the proof, we discuss the case of a single well potential

$$
W_{R}(|x|)=\chi(x / R) / R^{2}
$$

If we label the spherical harmonics in the standard way, $\phi_{l m}$, where $\Delta_{S^{n-1}} \phi_{l m}=$ $l(l+n-2) \phi_{l m}$, any solution $u, u_{f}$ to $(4.1)$ on $B_{0}(R)$ can be written

$$
u_{f}(r \omega)=\sum_{|m| \leq l, l=0}^{\infty} a_{l m} r^{-(n-2) / 2} J_{l+(n-2) / 2}(k r) \phi_{l m}(\omega),
$$

where $J_{\nu}$ is the standard Bessel function of order $\nu$, and

$$
u(r \omega)=\sum_{|m| \leq l, l=0}^{\infty} b_{l m} r^{-(n-2) / 2} \mathcal{J}_{l, k, \lambda, R}(r) \phi_{l m}(\omega),
$$

where $\mathcal{J}_{l, k, \lambda, R}(r)$ is the unique solution to

$$
\left(-\partial_{r}^{2}-\frac{1}{r} \partial_{r}+\frac{(l+(n-2) / 2)^{2}}{r^{2}}-\lambda W_{R}(r)-k^{2}\right) \mathcal{J}_{l, k, \lambda, R}(r)=0,
$$

which is equal to $J_{l+(n-2) / 2}\left(\sqrt{\lambda / R^{2}+k^{2}} r\right)$ near $r=0$. (This is, up to scale, the unique regular solution, since $W_{R}=1 / R^{2}$ near $r=0$.)

From this it is clear that a necessary and sufficient condition for solving both (4.1) and (4.2) is that there exist a non-negative integer $l$ and a $k>0$ such that the Wronskian

$$
D_{l, k, \lambda, R}(r):=\operatorname{det}\left(\begin{array}{cc}
J_{l+(n-2) / 2}(k r) & \mathcal{J}_{l, k, \lambda, R}(r) \\
\partial_{r}\left(J_{l+(n-2) / 2}(k r)\right) & \partial_{r}\left(\mathcal{J}_{l, k, \lambda, R}(r)\right)
\end{array}\right)
$$


satisfies

$$
D_{l, k, \lambda, R}(R)=0
$$

We will prove

Lemma 2. For fixed $l, \lambda$, and $R$, the set of zeroes of $D_{l, k, \lambda, R}(R)$ as a function of $k$ is discrete (hence countable) in $(0, \infty)$. For fixed $l, \lambda$, and $k$, the set of zeroes of $D_{l, k, \lambda, R}(R)$ as a function of $R$ is discrete (hence countable) in $(0, \infty)$.

Assuming the lemma, the theorem follows easily.

Proof of Theorem 1. Fix a sequence $\lambda_{i} \rightarrow \infty$, and a particular element $\lambda$, thereof. By the proceeding discussion, given any $R<\left|x_{0}\right|-1$, there is a solution to (4.1) and (4.2) on $\Omega_{R}$ if and only if there are $l, \tilde{l} \geq 0$, and $k>0$ so that we have both

$$
\begin{gathered}
D_{l, k, \lambda, R}(R)=0, \\
D_{\widetilde{l}, k, \lambda, 1}(1)=0 .
\end{gathered}
$$

Fixing $l, \widetilde{l}$, consider the set of $R>0$ for which there is a solution to (4.9). Using the first part of Lemma 2 , there are only countably many solutions, $k_{i}$, to the second equation in (4.9). For each $k_{i}$, using the second part of Lemma 2, there are only countably many solutions $R_{i, j}$ to $D_{l, k_{i}, \lambda, R_{i, j}}\left(R_{i, j}\right)=0$, and thus there are only countably many $R$ for which the system (4.9) admits a solution. There are only countably many pairs $(l, \widetilde{l})$ of non-negative integers, so the set of $R$ such that $(4.9)$ holds for any pair $(l, \widetilde{l})$ is countable.

Thus, for each $\lambda_{i}$, there are no solutions to (4.9) with $l, \tilde{l} \in\{0,1,2, \ldots\}$ and $R<$ $\left|x_{0}\right|-1$ not in some countable set $\Lambda_{i}$. Setting $\Lambda=\bigcup_{i} \Lambda_{i}$ proves the theorem.

It remains to prove the lemma.

Proof of Lemma 2. To simplify notation, for fixed $R, l$, and $\lambda$ set $F(k):=D_{l, k, \lambda, R}(R)$, and for fixed $k, l$, and $\lambda$, set $G(R)=D_{l, k, \lambda, R}(R)$. Our goal is to prove that both $F(k)$ and $G(R)$ have at most countably many zeroes. To see this, note that by scaling the $r$ variable in $(4.6), \mathcal{J}_{l, k, \lambda, R}(r)=f(l, k R, \lambda, r / R)$, where $f$ satisfies the differential equation

$$
\left(-\partial_{r}^{2}-\frac{1}{r} \partial_{r}+\frac{(l+(n-2) / 2)^{2}}{r^{2}}-\lambda W_{1}(r)-k^{2}\right) f(l, k, \lambda, r)=0 .
$$

The two terms in $D_{l, k, \lambda, R}(R)$ involving $\mathcal{J}_{l, k, \lambda, R}$ are, $\mathcal{J}_{l, k, \lambda, R}(R)=f(l, k R, \lambda, 1)$ and $\left.\partial_{r}\left(\mathcal{J}_{l, k, \lambda, R}(r)\right)\right|_{r=R}=\partial_{r} f(l, k R, \lambda, 1) / R$. These are analytic functions of $k R$ by Theorem XI.56 in [10]. Bessel functions are analytic, so $D_{l, k, \lambda, R}(R)$ is analytic in both $k$ and $R$.

It therefore suffices to check that neither $F(k)$ nor $G(R)$ is identically zero, which we do using the Sturm comparison theorem and taking $k$ and $R$ large in comparison with $\lambda$. Note that $D_{l, k, \lambda, R}(R)$ is non-zero precisely when $\mathcal{J}_{l, k, \lambda, R}$ is not a multiple of $J_{l+(n-2) / 2}$ for $r \geq R / 2$. We show this is true for when $k R$ is large by comparing $\mathcal{J}_{l, k, \lambda, R}$ to the solutions to equation (4.6) when the potential $-\lambda W_{R}$ is replaced by either $-\lambda R^{-2} 1_{[0, R / 4]}$ or $-\lambda R^{-2} 1_{[0, R / 2]}$. Since $R^{-2} 1_{[0, R / 4]} \leq W_{R} \leq R^{-2} 1_{[0, R / 2]}$, the Sturm comparison theorem tells us that the $N$ th zero of $\mathcal{J}_{l, k, \lambda, R}$ is between that of the solution to equation (4.6) with $W_{R}$ replaced with the characteristic functions 


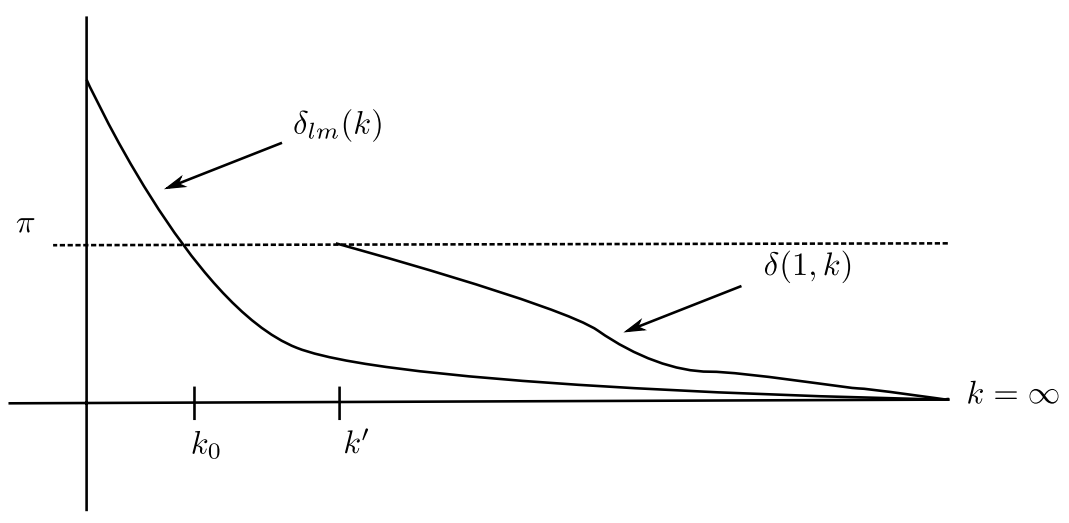

Figure 2. For $k$ large, the phase shifts are monotone in $s$.

above. Since the solutions for these are $J_{l+(n-2) / 2}\left(\sqrt{k^{2}+\lambda R^{-2}} r\right)$ on the support of the characteristic function, and since $\sqrt{k^{2}+\lambda R^{-2}}=k+O\left(1 /\left(k R^{2}\right)\right)$, we find that the zeroes of $\mathcal{J}_{l, k, \lambda, R}(r)$ for $r \geq R$, which are $\sim \pi / k$ apart, are shifted by an amount between $c_{1} k^{-2} R^{-1}$ and $c_{2} k^{-2} R^{-1}$ relative to those of $J_{l+(n-2) / 2}(k r)$, where $0<c_{1}<c_{2}$. This shows that when $k$ is large for fixed $R$, or for $R$ large for fixed $k$, then $\mathcal{J}_{l, k, \lambda, R}(r)$ is not equal to $J_{l+(n-2) / 2}(k r)$ for $r \geq R$ and hence that $D_{l, k, \lambda, R}(R) \neq 0$.

\section{Monotonicity of phase shifts and the existence of almost partially transparent frequencies}

For any potential $V \geq 0$, it is not difficult to show, using the monotonicity result of [4], that the number of non-zero almost partially transparent frequencies of $\Delta-\lambda V$ is unbounded as $\lambda \rightarrow \infty$. We sketch an argument now. Without loss of generality, assume that $V(0) \neq 0$, and let $\chi=\chi(r) \in C_{c}^{\infty}$ be a smooth, non-negative, sphericallysymmetric function with $V \geq \chi$. Setting $V(s):=s V+(1-s) \chi$, let $S_{s}(k)$ be the scattering matrix for $\Delta-\lambda V(s)$ at frequency $k$. Let $\alpha(s, k)=\exp (2 i \delta(s, k))$ be an eigenvalue of $S_{s}(k)$. As long as it is not equal to $1, \alpha(s, k)$ can be taken analytic in $s$. If $\alpha(s, k) \neq 1$ for all $s \in[0,1]$, Theorem 1 of [4] gives

$$
\frac{\partial \delta}{\partial s} \geq 0 \text { for } s \in[0,1]
$$

Recall that for $s=0$, i.e., for the potential $-\lambda \chi$, the scattering matrix is diagonal with respect to (say, the standard) basis of spherical harmonics, $\phi_{l m}$. The eigenvalues $\alpha_{l m}(k)=\exp \left(2 \mathrm{i} \delta_{l m}(k)\right)$ are defined continuously for all $k$, and can be taken so that $\delta_{l m}(k) \rightarrow 0$ as $k \rightarrow \infty$. As we saw in our discussion of Levinson's theorem for central potentials in the introduction, by taking $\lambda$ sufficiently large, the counterclockwise winding number of $\alpha_{l m}(k)$ can be assumed bigger than 1. For $k$ taken large so that $S_{s}(k)$ is very close to the identity, let $\alpha(s, k)$ be an eigenvalue with $\alpha(0, k)=\alpha_{l m}(k)$. By $(5.1), \delta_{l m}(k)<\delta(1, k)$. If $\lambda$ was chosen large enough so that $\delta_{l m}(k)$ crosses $\pi$, say at $k_{0}$, then $\delta(1, k)$ must approach $\pi$ from below at some frequency $k^{\prime} \geq k_{0}$ - see figure 2. This produces a non-zero almost partially transparent eigenvalue for each pair $(l, m)$ for which the winding number of $\alpha_{l m}(k)$ is bigger than 1. 
As a simple corollary to this argument we have

Corollary 3. For $V_{R}$ and $\lambda_{i} \rightarrow \infty$ as in Theorem 1, and for $\lambda_{i}$ large enough, there exist eigenvalues of the scattering matrix $S_{\lambda_{i} V_{R}}$ which approach 1 at non-zero frequencies. Since $-\lambda_{i} V_{R}$ is completely non-transparent, these limiting frequencies are almost partially transparent but not partially transparent.

\section{On Newton's "micro Levinson theorem" for non-central potentials}

In 1989, R. Newton published a paper The spectrum of the Schrödinger S matrix: low energies and a new Levinson theorem [8], claiming the following result:

Claimed Theorem. Assume that the potential [is smooth and exponentially decaying]. Then each eigenphase shift $\delta_{l m}(k)$ may be defined to be a continuous function of $k$, to vanish at $k \rightarrow \infty$, and so that its value at the origin is $\delta_{l m}(0)=\pi\left(\mathcal{N}_{l m}+\nu\right)$ where $\mathcal{N}_{l m}$ is the number of bound states associated with the pair $(l, m), \nu=1 / 2$ if $l=0$ and there is a half-bound state, and $\nu=0$ otherwise.

To label bound states, Newton introduces a strength parameter $\lambda$ and considers the negative spectrum for the family $\lambda V$; as $\lambda \rightarrow 0$ the bound states approach zero energy and then disappear, and the label is related to the asymptotic spatial behaviour of the limiting zero energy solution.

Corollary 3 above shows that Newton's claimed theorem is incorrect. Indeed, for $V_{R}$ and $\lambda_{i}$ as in the corollary, there are eigenvalues of the scattering matrix of $\Delta-\lambda_{i} V$ which approach 1 as $k \downarrow k_{0}$ for some non-zero frequency $k_{0}$, but such that 1 is not an eigenvalue of $S\left(k_{0}\right)$. Therefore, the phase shifts of $S(k)$ cannot always be taken continuous on $k \in(0, \infty)$.

We mention some other problems with Newton's paper:

- The proposed labelling of bound states is flawed. It seems to depend implicitly on the assumption that the limiting eigenfunctions of the scattering matrix $S_{\lambda V}(k)$ as $k \rightarrow 0$ is independent of $\lambda$, but this is not the case.

- Lemma 4.2 of [8] is incorrect. Using the notation in the lemma, if $u$ is a zero energy bound state of $(\Delta+V) u=0$ with angular dependence $r^{-l-1} \mathcal{Y}_{l n}(\omega)$, where $\mathcal{Y}_{l n}(\omega)$ is a spherical harmonic with angular momentum quantum number $l$, then $u=K u$ and $P_{l n} u=u$, and hence $u=K P_{l n} u$. However, the converse is certainly not true: from $u=K P_{l n} u$ we are not able to deduce that both $u=K u$ and $u=P_{l n} u$, which would be required to conclude Lemma 4.2.

The second point is of some significance: if Lemma 4.2 were correct for every $C_{c}^{\infty}\left(\mathbb{R}^{3}\right)$ potential $V$, there would be infinitely many $\lambda$ such that $\Delta-\lambda V$ had a zero eigenvalue with eigenfunction behaving as $r^{-l-1} \mathcal{Y}_{l n}(\omega)$ at infinity, for some spherical harmonic $\mathcal{Y}_{l n}(\omega)$ (depending on $\lambda$ ) with angular momentum quantum number $l$. However, we are sure this is not the case. In fact, we conjecture that the generic potential $V \in C_{c}^{\infty}\left(\mathbb{R}^{n}\right)$ is such that all the zero energy eigenfunctions of $\Delta+\lambda V$ are half-bound states. 


\section{Open problems}

This short note suggests some interesting open problems concerning potential scattering:

- Is the generic potential well in $C_{c}^{\infty}\left(\mathbb{R}^{n}\right)$ completely non-transparent?

- Is it true that for a generic potential $V$ in $C_{c}^{\infty}\left(\mathbb{R}^{n}\right)$, all the zero-energy, decaying solutions $u$ to $(\Delta-\lambda V) u=0$ are half-bound states? That is, are $L^{2}$ zero-energy eigenfunctions nongeneric relative to half-bound states?

- Is there a Levinson-type theorem for non-central potentials where instead of looking at the value of phase-shifts at $k=0$, we count the number of almost partially transparent energies (counted with multiplicity)?

- Can one characterize, in some spectral-geometric way, the almost partially transparent frequencies of a potential well $-V \in C_{c}^{\infty}\left(\mathbb{R}^{n}\right)$, by analogy with that for obstacle scattering [2]?

\section{Acknowledgments}

The authors would like to thank Lennie Friedlander, Rafe Mazzeo and Yuri Safarov for helpful conversations during the writing of this paper.

\section{References}

[1] A. H. Barnett and A. Hassell, Fast computation of high frequency Dirichlet eigenmodes via the spectral flow of the interior Neumann-to-Dirichlet map, Comm. Pure Appl. Math., to appear; arXiv: 1112.5665 .

[2] J. -P. Eckmann and C. -A. Pillet, Spectral duality for planar billiards, Comm. Math. Phys. 170(2) (1995), 283-313.

[3] L. Friedlander, Some inequalities between Dirichlet and Neumann eigenvalues, Arch. Rational Mech. Anal. 116(2) (1991), 153-160.

[4] J. W. Helton and J. V. Ralston, The first variation of the scattering matrix, J. Differ. Equ. 21(2) (1976), 378-394.

[5] L. Hörmander, The analysis of linear partial differential operators III, Springer, Berlin, 1985.

[6] R. Melrose, Geometric scattering theory, Cambridge University Press, Cambridge, 1995, ISBN 0-521-49673-X.

[7] R. G. Newton, Analytic properties of radial wave functions, J. Math. Phys. 1 (1960), 319-347;

- Analytic properties of radial wave functions, J. Math. Phys. 1 (1960), errata, 452.

[8] - The spectrum of the Schrödinger $S$ matrix: low energies and a new Levinson theorem, Ann. Phys. 194(1) (1989), 173-196.

[9] M. Reed and B. Simon, Methods of modern mathematical physics. IIII, Academic Press, New York, 1978, ISBN 0-12-585004-2.

[10] - Methods of modern mathematical physics. III, Academic Press, New York, 1979, ISBN 0-12-585003-4.

[11] D. Yafaev, Mathematical scattering theory: analytic theory, American Mathematical Society, Providence, RI, 2010, ISBN 978-0-8218-0331-8.

Department of Mathematics, Australian National University, Canberra ACT 0200, AusTRALIA

E-mail address: Andrew.Hassell@anu.edu.au

Department of Mathematics, Australian National University, Canberra ACt 0200, AusTRALIA

E-mail address: Jesse.Gell-Redman@anu.edu.au 
